\title{
Valoración radiológica y manejo quirúrgico de una interrupción del arco aórtico, a propósito de un caso
}

\section{Radiological evaluation and surgical management of an aortic arch interruption, on the subject of a case}

\author{
Alejandro Vallecillo-Torres ${ }^{1 *}$, Andrés Soto-Rodríguez ${ }^{2}$ y Sharon Mendoza-Solís ${ }^{3}$ \\ ${ }^{1}$ Servicio de Radiología, Hospital San Juan de Dios; ${ }^{2}$ Unidad de Investigación, Hospital San Juan de Dios; ${ }^{3}$ Servicio de Cirugía Cardiovascular, \\ Hospital Nacional de Niños. Caja Costarricense de Seguro Social, San José, Costa Rica
}

\section{Resumen}

La interrupción del arco aórtico es una malformación vascular congénita poco común. Consiste en la ausencia de fusión entre la aorta ascendente y la descendente. Esta enfermedad a menudo se asocia con otras malformaciones cardiacas, como comunicación interventricular y conducto arterioso permeable. La valoración radiológica ayuda no solo al diagnóstico, sino también a la planeación de la corrección quirúrgica más adecuada. Debido a su poca frecuencia, el médico tratante puede necesitar algún tipo de apoyo para el requerimiento diagnóstico necesario para el abordaje de esta patología por los cirujanos. La radiografía de tórax, el ecocardiograma, la tomografía computarizada y la resonancia magnética son técnicas adecuadas para el diagnóstico de esta patología. Cada una de ellas tiene signos específicos que se deben conocer para lograr la mayor cantidad de información a beneficio del paciente.

Palabras clave: Aorta. Enfermedades de la aorta. Anomalías congénitas. Pediatría.

\begin{abstract}
Aortic arch interruption is a rare congenital vascular malformation. This pathology consists of an absence of fusion between the ascending and descending aorta. This disease is often associated with other cardiac malformations such as ventricular septal defect and a patent ductus arteriosus. The radiological evaluation helps, not only in the diagnosis but also in the planning of the most appropriate surgical correction. Due to the infrequency of this entity, the treating physician may need some type of support for the diagnostic requirements necessary for the approach of this pathology by the surgeons. Chest radiography, echocardiography, computed tomography, and magnetic resonance imaging are suitable techniques for the diagnosis of this pathology. Each of these has specific signs that must be known to obtain the greatest amount of information for the benefit of the patient.
\end{abstract}

Key words: Aorta. Aortic diseases. Congenital abnormalities. Pediatrics.

Correspondencia:

*Alejandro Vallecillo-Torres

E-mail: dr.vallecillo.torres@gmail.com
Disponible en internet: 03-12-2021

Rev Chil Radiol. 2021;27(4):210-213

www.resochradi.com

0717-9308 / @ 2021 Sociedad Chilena de Radiología. Publicado por Permanyer. Éste es un artículo open access bajo la licencia CC BY-NC-ND (https://creativecommons.org/licenses/by-nc-nd/4.0/). 


\section{Introducción}

La interrupción del arco aórtico (IAA) es una malformación cardiovascular poco frecuente, en la que existe una interrupción anatómica propiamente del arco aórtico y su continuidad entre la aorta ascendente y la descendente ${ }^{1}$. Este defecto puede ser total o estar conectado por una banda fibrosa remanente. Con frecuencia se presentan una comunicación interventricular (CIV) o un conducto arterioso permeable, como se muestra en la figura $1^{2}$.

Esta patología se presenta en el 1\% de las cardiopatías congénitas infantiles, con una incidencia de 19 casos por millón de nacidos vivos ${ }^{3,4}$. Cerca del $50 \%$ de los pacientes con IAA, también llamado síndrome de DiGeorge, presentan una anomalía cromosómica, específicamente una deleción de un pequeño segmento en 22q11.25.

Según Celoria y Patton $^{6}$, la IAA se puede clasificar en tres tipos de acuerdo con la ubicación del defecto:

- Tipo A: la interrupción anatómica ocurre distal al origen de la arteria subclavia izquierda. Este tipo de IAA corresponde en prevalencia a la segunda alteración más frecuentemente observada.

- Tipo B: el defecto anatómico se produce entre la carótida común izquierda y los orígenes de la arteria subclavia izquierda, y constituye la manifestación más común de la enfermedad (> 50\%).

- Tipo C: es la menos frecuente y en ella la interrupción ocurre proximal al origen de la carótida común izquierda.

El tratamiento definitivo de la IAA consiste en una intervención quirúrgica para la conexión de ambos segmentos de aorta. Una planificación adecuada del procedimiento requiere una evaluación de diagnóstico por imagen precisa para caracterizar correctamente la anatomía aórtica y cardiaca, y definir el tipo exacto de IAA. La pérdida de alguna estructura anatómica, así como una cuantificación errónea del defecto, pueden traer inconvenientes en el momento de la cirugía.

\section{Caso clínico}

Paciente nacida en el servicio de ginecología de un hospital provincial de Costa Rica, producto de embarazo único, segunda gesta $\left(G_{2}\right)$ de una madre sana de 26 años, sin patología prenatal reportada. La paciente nace por vía vaginal, producto de un parto a término a las 39 semanas de edad gestacional, con peso de $3350 \mathrm{~g}$, talla de $50 \mathrm{~cm}$ y Apgar 6/8. Requiere medidas de soporte por cianosis, dificultad respiratoria y

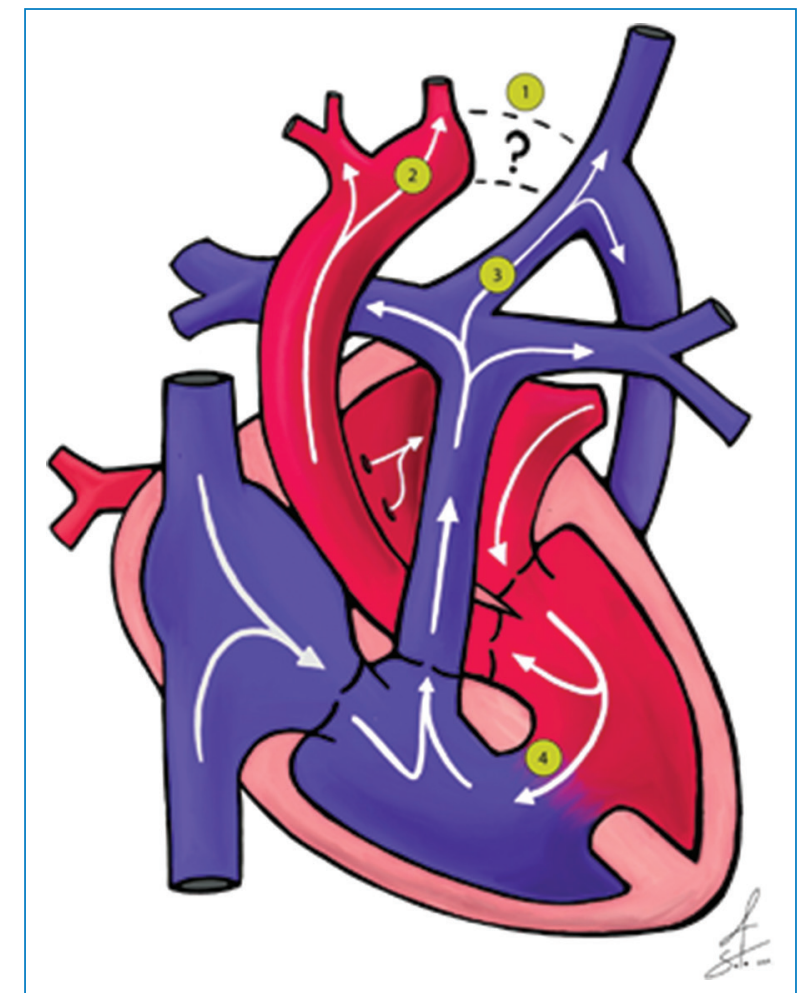

Figura 1. Defectos anatómicos presentes en una interrupción de arco aórtico. 1: interrupción del arco aórtico. 2: localización más común de interrupción del arco aórtico. 3: conducto arterioso permeable. 4: comunicación interventricular.

quejido, y es internada en la unidad de cuidados intensivos neonatales (UCIN) del mismo hospital.

Al efectuar la exploración física se identifica un soplo sistólico 2/6 plurifocal, por lo que se solicitan más estudios. En el ecocardiograma se documentan transposición de grandes arterias con CIV, comunicación interauricular (CIA) tipo ostium secundum y conducto arterioso permeable. Debido a esto, se traslada a la UCIN del Hospital Nacional de Niños - Caja Costarricense de Seguro Social.

Durante su internamiento se solicita un nuevo ecocardiograma, que solo documenta una CIV perimembranosa de $10 \mathrm{~mm}$ y una CIA de $4 \mathrm{~mm}$ con flujo de izquierda a derecha, y una imagen que se describe como sospechosa por coartación de aorta o interrupción de arco aórtico. Debido a estos hallazgos se solicita una angiografía por tomografía computarizada prospectiva mediante monitorización con electrocardiograma para disminuir los artefactos generados por el ciclo cardiaco y la respiración, en la cual se confirma una interrupción del arco aórtico (Fig. 2). 


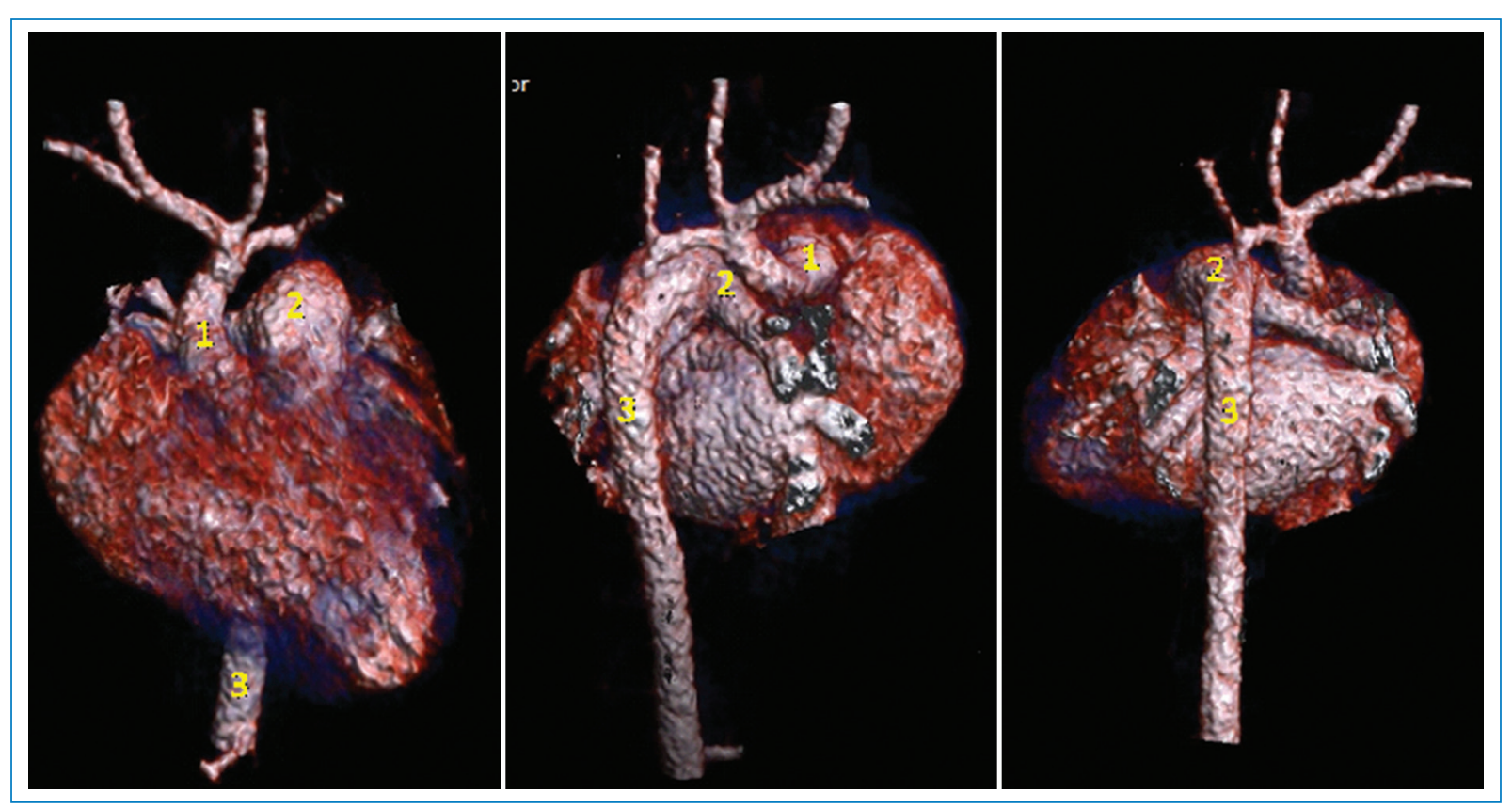

Figura 2. Reconstrucción por medio de tomografía computarizada del defecto de aorta presentado por la paciente del caso. 1: aorta ascendente. 2: restos de cayado aórtico. 3: aorta descendente.

Se lleva a sala de operaciones a los 62 días de haber nacido y durante la cirugía se coloca a la paciente en circulación extracorpórea. Los hallazgos operatorios confirman una interrupción del arco tipo $\mathrm{B}$, la transposición de los grandes vasos, una CIV y una CIA. Para la reparación se realiza una plastia de arco aórtico con parche de pericardio autólogo, cierre de la CIV con parche de Gore-Tex, switch arterial de Jatene y cierre primario de la $\mathrm{ClA}$. Durante la operación presentó un sangrado mediastinal que requirió una reintervención inmediata. La paciente es llevada a la UCIN, donde presenta múltiples complicaciones, entre ellas mediastinitis, peritonitis con Candida e insuficiencia renal aguda que requirió diálisis.

A los 14 días de la cirugía se realiza un ecocardiograma de control que demuestra una CIV remanente de $6 \mathrm{~mm}$ en la zona de implantación del parche, por lo que se decide llevarla al laboratorio de cateterismo, donde se documenta una insuficiencia aórtica grave y se decide reintervenir quirúrgicamente. Durante la operación surgen complicaciones, las cuales causan el lamentable fallecimiento de la paciente.

\section{Discusión}

Se han descrito diversos métodos para la evaluación radiológica de pacientes con sospecha de IAA, cada uno con beneficios y desventajas particulares, por lo cual es conveniente valorar cada paciente de forma individual.

Las principales características anatómicas que se deben identificar son la ubicación espacial y temporal, la longitud del defecto vascular aórtico, el calibre de la aorta torácica proximal y distal a la interrupción, el patrón de ramificación y los orígenes de los grandes vasos, la ubicación y la permeabilidad del conducto arterioso, la apariencia de los tractos de salida ventriculares y la presencia de cualquier otra anomalía cardiovascular?

La radiografía simple de tórax generalmente no es una técnica específica, pero suele servir para guiar el diagnóstico. Un botón aórtico ausente y una cardiomegalia son hallazgos que podrían estar relacionados con una posible IAA ${ }^{8}$.

La ecocardiografía se considera la técnica de imagen principal para el estudio de la IAA; su portabilidad y la no emisión de radiación ionizante ofrecen ventajas sobre otras tecnologías ${ }^{9,10}$. Durante este estudio, y para esta patología particular, el ventrículo derecho puede aparecer mucho más grande que el izquierdo, siendo este es un hallazgo inespecífico. La aorta ascendente, a su vez, puede aparecer en una posición de mayor verticalidad. Sin embargo, la visualización de la IAA no solo establece el diagnostico, sino que también sirve para tomar medidas de los defectos y describir la anatomía adyacente ${ }^{11}$. Este estudio tiene sus limitaciones, ya que este depende de la anatomía del 
paciente, las ventanas de exploración sonográficas y la experiencia del cardiólogo, y no siempre es posible examinar el arco aórtico en toda su extensión.

También se ha descrito el uso de la angiografía por tomografía computarizada no invasiva en la evaluación de la $I A A^{12,13}$, ya que permite visualizar el arco aórtico interrumpido en toda su extensión y las anomalías congénitas asociadas ${ }^{14}$. Existe la posibilidad de sincronizar el estudio electrocardiográficamente, generando un aumento en la resolución espacial y dando información de la función ventricular, tanto de las cámaras izquierdas como de las derechas ${ }^{15}$. Sin embargo, las consideraciones sobre la exposición a la radiación han limitado su aplicabilidad clínica, restringiendo su uso a ciertas circunstancias, como cuando no hay acceso a la ecocardiografía ni a la resonancia magnética (RM).

La RM, incluida la angiografía por RM, se ha descrito como una técnica útil para evaluar los defectos cardiacos complejos, como la IAA ${ }^{7}$. Esta técnica puede caracterizar de manera precisa la anatomía cardiovascular, incluida la de la aorta torácica y los grandes vasos, y las anomalías cardiacas coexistentes. Además, la RM también puede proporcionar información útil sobre la función de la cámara y la válvula cardiacas. En otros países esta técnica se considera el método de referencia para el diagnóstico de la IAA. En nuestro medio, el acceso a la RM es limitado, además de que implica el traslado de un paciente crítico a otro centro hospitalario, por lo que no se favorece su utilización sistemática.

\section{Conclusiones}

Las imágenes médicas son de gran importancia para el diagnóstico y el tratamiento de las cardiopatías congénitas. Elegir la técnica correcta y estandarizar la manera en que se reportan las patologías ayuda a realizar un diagnóstico certero y a planear intervenciones quirúrgicas más precisas.

\section{Agradecimientos}

El equipo investigador quiere agradecer al Dr. Alfredo Sanabria-Castro y a la Dra. Ann Echeverri-McCandlesss por el apoyo en el momento de la redacción del artículo.

\section{Financiamiento}

El financiamiento de este estudio fue por cuenta propia de los investigadores.

\section{Conflicto de intereses}

Ninguno de los autores presenta conflictos de intereses.

\section{Responsabilidades éticas}

Protección de personas y animales. Los autores declaran que para esta investigación no se han realizado experimentos en seres humanos ni en animales.

Confidencialidad de los datos. Los autores declaran que han seguido los protocolos de su centro de trabajo sobre la publicación de datos de pacientes.

Derecho a la privacidad y consentimiento informado. Los autores declaran que en este artículo no aparecen datos de pacientes.

La presente iniciativa se desarrolló en apego a los tratados internacionales de bioética; respetando la normativa nacional vigente y su desarrollo fue de conocimiento por el Área de Bioética de la CCSS, cuenta con su respectivo aval de publicación e igualmente los autores se comprometen a adherirse a las normas éticas de la Revista Chilena de Radiología.

\section{Bibliografía}

1. Ramírez Alcántara J, Méndez MD. En: StatPearls [Internet]. Treasure Island (FL): StatPearls Publishing; 2021 Jan. Disponible en: https://www. ncbi.nlm.nih.gov/books/NBK532902/.

2. Tang $X$, Wang $L$, Wu $Q$, Tong $X$. Persistent fifth aortic arch with interrupted aortic arch. J Card Surg. 2015;30:284-7.

3. Zhu $P, Y e X$, Zhao Q. Successful surgical treatment of descending aorta interruption: a case report. Heart Surg Forum. 2018;21:E175-6.

4. Wang P, Wu SP, Jiang R, Du FZ. 320-slice CT angiography of an interrupted aortic arch patient relying on collaterals: an addition to classification criteria. Radiol Case Rep. 2019;14:1382-4.

5. Momma K. Cardiovascular anomalies associated with chromosome 22q11.2 deletion syndrome. Am J Cardiol. 2010;105:1617-24.

6. Celoria GC, Patton RB. Congenital absence of the aortic arch. Am Heart J. 1959;58:407-13.

7. Dillman JR, Yarram SG, D'Amico AR, Hernandez RJ. Interrupted aortic arch: spectrum of MRI findings. Am J Roentgenol. 2008;190:1467-74.

8. Jaffe RB. Complete interruption of the aortic arch 1. Characteristic radiographic findings in 21 patients. Circulation. 1975;52:714-21.

9. Kaulitz R, Jonas RA, van der Velde ME. Echocardiographic assessment of interrupted aortic arch. Cardiol Young. 1999:9:562-71.

10. Teo E-LHJ, Goldberg CS, Strouse PJ, Vermilion RP, Bove EL. Aortopulmonary window with interrupted aortic arch and pulmonary artery sling: diagnosis by echocardiography and magnetic resonance imaging: case report and literature review. Echocardiography. 1999;16:147-50.

11. Goudar SP, Shah SS, Shirali GS. Echocardiography of coarctation of the aorta, aortic arch hypoplasia, and arch interruption: strategies for evaluation of the aortic arch. Cardiol Young. 2016;26:1553-62.

12. Cinar A, Haliloglu M, Karagoz T, Karcaaltincaba M, Celiker A, Tekinalp G. Interrupted aortic arch in a neonate: multidetector CT diagnosis. Pediatr Radiol. 2004;34:901-3.

13. Wong MNL, Chan LG, Sim KH. Interrupted aortic arch and aortopulmonary window demonstrated on 64-slice multidetector computed tomography angiography. BMJ Case Rep. 2009;2009:bcr2006088898.

14. Hanneman K, Newman B, Chan F. Congenital variants and anomalies of the aortic arch. RadioGraphics. 2017;37:32-51.

15. Desjardins B, Kazerooni EA. ECG-gated cardiac CT. Am J Roentgenol. 2004;182:993-1010. 